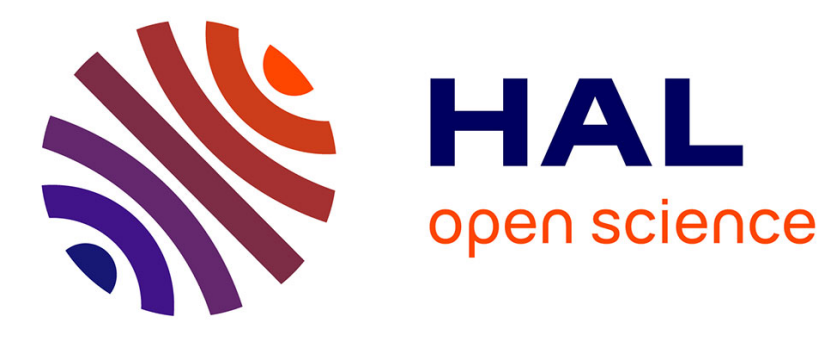

\title{
Polynomial modal analysis of slanted lamellar gratings
}

Gérard Granet, Manjakavola Honoré Randriamihaja, Karyl Raniriharinosy

\section{To cite this version:}

Gérard Granet, Manjakavola Honoré Randriamihaja, Karyl Raniriharinosy. Polynomial modal analysis of slanted lamellar gratings. Journal of the Optical Society of America. A Optics, Image Science, and Vision, 2017, 34 (6), pp.975-982. 10.1364/JOSAA.34.000975 . hal-01525559

\section{HAL Id: hal-01525559 \\ https://hal.science/hal-01525559}

Submitted on 21 May 2017

HAL is a multi-disciplinary open access archive for the deposit and dissemination of scientific research documents, whether they are published or not. The documents may come from teaching and research institutions in France or abroad, or from public or private research centers.
L'archive ouverte pluridisciplinaire HAL, est destinée au dépôt et à la diffusion de documents scientifiques de niveau recherche, publiés ou non, émanant des établissements d'enseignement et de recherche français ou étrangers, des laboratoires publics ou privés. 


\title{
Polynomial modal analysis of slanted lamellar gratings
}

\author{
Gérard Granet ${ }^{1}$, \\ Manjakavola Honore Randriamihaja, ${ }^{1,2}$, Karyl Raniriharinosy ${ }^{2}$ \\ ${ }^{1}$ Université Clermont Auvergne, CNRS, SIGMA Clermont, \\ Institut Pascal, F-63000 Clermont-Ferrand, France \\ ${ }^{2}$ Lapauf,Université de Fianarantsoa,B.P.1264 Madagascar
}

\begin{abstract}
The problem of diffraction by slanted lamellar dielectric and metallic gratings in classical mounting is formulated as an eigenvalue eigenvector problem. The numerical solution is obtained by using the moment method with Legendre polynomials as expansion and test functions which allows to enforce in an exact manner the boundary conditions which determine the eigen-solutions. Our method is successfully validated by comparison with other methods including in the case of highly slanted gratings.
\end{abstract}

Index terms - Diffraction and gratings, Computational electromagnetic methods, Electromagnetic optics.

\section{Introduction}

Diffraction gratings and other periodic structures play important roles in photonics [1][2]. Slanted lamellar gratings are a particular example of such structures. They have application for blazing or for in and out coupling of light. Here, we are concerned with the electromagnetic analysis of dielectric and metallic gratings with slanted walls. Slanted lamellar grating have previously been treated using the rigorous coupled wave approach [3] [4] the fast Fourier factorization method [5] or a coordinate transformation method [6] [7]. More recently, Campbell et al developed the Differential modal method (DMM)[8] where a slanted lamellar grating is considered as a stack of $N$ individual lamellar gratings whose modes are coupled using a Bloch mode-matching technique. In this paper, we develop a modal method for diffraction by slanted lamellar gratings. First, as was done in [6], a coordinate transformation is introduced in the grating region so that the lamellar structure becomes translation invariant along one direction which also means that the dielectric constant depends only on one spatial coordinate. It becomes then straightforward to give the problem a modal formulation. Many methods exist to solve it numerically. The accuracy of the solution is linked with the treatment of the transverse boundary conditions that 
the electromagnetic field has to satisfy at the interface between the different dielectrics or metals. Using polynomials expansions within each domain of the lamellar gratings allows to express rigorously the different continuity relations that determine the eigenvalue problem and thus leads to exponential convergence for the eigenvalues and eigenvectors as was shown by Morf [9]. Recently, the polynomial modal method was revisited and Gegenbaueur polynomials were introduced [10][11] whereas Morf only used Legendre and Tchebycheff polynomials. The case of conical incidence was also investigated in [12] using the same approach as Morf. The goal of the present paper is to generalize the use of polynomial expansions for slanted lamellar gratings. The paper is organized

as follows: in section 2, we present the slanted lamellar grating problem and its spectral formulation in an inclined coordinate system. In section 3 , by using the Galerkin method with Legendre polynomial expansion, we derive the matrix eigenvalue equation. In our formulation, the polarization is taken into account thanks to a connection matrix which expresses explicitly the transverse boundary conditions. In section 4 , we validate our method by comparison with already published data.

\section{Presentation of the problem}

The slanted lamellar grating diffraction problem is depicted in fig 1 . This structure is illuminated from an homogeneous medium with optical index $\nu_{1}$ by a monochromatic plane wave with a wavelength $\lambda$ and an angular frequency $\omega$. The wave vector $\boldsymbol{k}$ forms an angle $\theta$ with respect to the axis $y$ axis. Hence its Cartesian coordinates are $k_{x}=k \alpha_{0}=k \nu_{1} \sin (\theta)$ and $k_{y}=k \beta_{0}=-k \nu_{1} \cos (\theta)$ with $k=2 \pi / \lambda$ the wavenumber. The $\exp (i \omega t)$ time dependence will be omitted throughout this paper. The grating region separates the incident medium from a substrate with refractive index $\nu_{3}$. The grating layer with thickness $h$, is characterized by a piecewise homogeneous permittivity function $\epsilon(x, y)$ that is periodic in the $x$ direction with period $d$. The side walls of the different materials of the grating are inclined at an angle $\phi$ with respect to the vertical axis. In the Cartesian coordinates $(x, y)$ the permittivity function depends on the two variables $x$ and $y$. However it is easy to find a new coordinate system in which the side walls fit a coordinate surface. One such coordinate system $\left(x^{1}, x^{2}, x^{3}\right)$ is described by:

$$
x^{1}=x-\tan (\phi) y, \quad x^{2}=y, \quad x^{3}=z
$$

The Jacobian matrix of this transformation is:

$$
\boldsymbol{J}=\left(\begin{array}{lll}
\frac{\partial x}{\partial x^{1}} & \frac{\partial x}{\partial x^{2}} & \frac{\partial x}{\partial x^{3}} \\
\frac{\partial y}{\partial x^{1}} & \frac{\partial y}{\partial x^{2}} & \frac{\partial y}{\partial x^{3}} \\
\frac{\partial z}{\partial x^{1}} & \frac{\partial z}{\partial x^{2}} & \frac{\partial z}{\partial x^{3}}
\end{array}\right)=\left(\begin{array}{ccc}
1 & \tan (\phi) & 0 \\
0 & 1 & 0 \\
0 & 0 & 1
\end{array}\right)
$$

In this coordinate system, the location of the side walls are given in terms of 


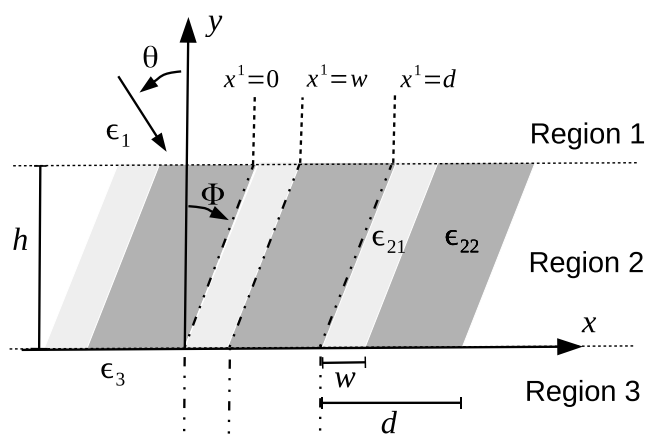

Figure 1: Geometry of the problem and illustration of the change of coordinates. $\epsilon_{1}=\nu_{1}^{2}, \epsilon_{3}=\nu_{3}^{2}$

the only $x^{1}$ variable. Indeed we have:

$$
\epsilon\left(x^{1}\right)= \begin{cases}\epsilon_{21} & \text { for } 0 \leq x^{1} \leq w \\ \epsilon_{22} & \text { for } w \leq x^{1} \leq d\end{cases}
$$

Thus, in the inclined coordinate system a binary grating fits two domains $\Omega_{1}=$ $\left\{x^{1}, 0 \leq x^{1} \leq w\right\}$ and $\Omega_{2}=\left\{x^{1}, w \leq x^{1} \leq d\right\}$ The metric tensor $g_{i j}$ of the new coordinate system is obtained from the Jacobian matrix $\boldsymbol{J}$ and its transpose $\boldsymbol{J}^{T}$ by:

$$
\left[g_{i j}\right]=\boldsymbol{J}^{T} \boldsymbol{J}
$$

from which we deduce the conjugate metric tensor $\left[g^{i j}\right]$ :

$$
\left[g^{i j}\right]=\left[g_{i j}\right]^{-1}=\left(\begin{array}{lll}
\frac{1}{\cos ^{2}(\phi)} & -\tan (\phi) & 0 \\
-\tan (\phi) & 1 & 0 \\
0 & 0 & 1
\end{array}\right)
$$

One could choose to solve the whole problem with the inclined coordinate system. As far as we are concerned, we restrict its use to the grating region. It has to be remarked that coordinates surfaces $y=h$ and $y=0$ are common to all coordinate systems and that the only Cartesian component of a vector field $\boldsymbol{A}$ affected by the change of coordinates is $A_{y}$. Indeed, the covariant components of $\boldsymbol{A}$ are written in terms of the Cartesian components as follows:

$$
A_{1}=A_{x}, \quad A_{3}=A_{z}, \quad A_{2}=\tan (\phi) A_{x}+A_{y}
$$

So, whatever the coordinate system, we are led to calculate components $A_{3}$ and $A_{1}$ in the three regions that define the problem and to match them at $y=h$ and $y=0$. 


\section{$3 \quad$ Theory}

\subsection{MAXWELL'S EQUATIONS}

In general curvilinear coordinates, with a time dependence of the form $\exp (i \omega t)$, it is convenient to use covariant Maxwell equations written with the Einstein convention as [13], [14]:

$$
\left\{\begin{array}{l}
\xi^{i j k} \partial_{j} E_{k}=-i \omega B^{i} \\
\xi^{i j k} \partial_{j} H_{k}=i \omega D^{i}
\end{array}\right.
$$

$\partial_{j}$ denotes the partial derivative operator with respect to variable $x^{j} \cdot \xi^{i j k}$ denotes the Lévi-Cività indicator whose non-null components are: $\xi^{1,2,3}=\xi^{2,3,1}=$ $\xi^{3,1,2}=1$ and $\xi^{1,3,2}=\xi^{2,1,3}=\xi^{3,2,1}=-1$. The geometry appears in the constitutive relations along with the material's physical properties:

$$
\left\{\begin{array}{l}
D^{i}=\epsilon_{0} \epsilon \sqrt{g} g^{i j} E_{j} \\
B^{i}=\mu_{0} \sqrt{g} g^{i j} H_{j}
\end{array}\right.
$$

where $\epsilon$ is the real or complex relative permittivity. Since the structure is translation invariant along the $x^{3}$ direction, we have $\partial_{3}=0$ and Maxwell's equations decouple into two sets of independent first-order differential equations corresponding to TE polarization and TM polarization:

TE polarization

$$
\begin{array}{r}
\partial_{2} E_{3}=-i \omega \mu_{0}\left(\sqrt{g} g^{11} H_{1}+\sqrt{g} g^{12} H_{2}\right) \\
-\partial_{1} E_{3}=-i \omega \mu_{0}\left(\sqrt{g} g^{21} H_{1}+\sqrt{g} g^{22} H_{2}\right) \\
\partial_{1} H_{2}-\partial_{2} H_{1}=i \omega \epsilon_{0} \epsilon E_{3}
\end{array}
$$

TM polarization

$$
\begin{array}{r}
\partial_{2} H_{3}=i \omega \epsilon_{0} \epsilon\left(\sqrt{g} g^{11} E_{1}+\sqrt{g} g^{12} E_{2}\right) \\
-\partial_{1} H_{3}=i \omega \epsilon 0\left(\sqrt{g} g^{21} E_{1}+\sqrt{g} g^{22} E_{2}\right) \\
\partial_{1} E_{2}-\partial_{2} E_{1}=-i \omega \mu_{0} H_{3}
\end{array}
$$

By eliminating $H_{2}$ in 11 and $E_{2}$ in 14 we get:

TE polarization

$$
\begin{aligned}
\partial_{2}\left[\begin{array}{l}
E_{3} \\
H_{1}
\end{array}\right]= & \\
& {\left[\begin{array}{cc}
\frac{-g^{12}}{g^{22}} \partial_{1} & -i \omega \mu_{0} \frac{1}{\sqrt{g} g^{22}} \\
-i \omega \epsilon_{0}\left(\epsilon\left(x^{1}\right) \sqrt{g} g^{33}+\frac{1}{k^{2}} \partial_{1} \frac{1}{\sqrt{g} g^{22}} \partial_{1}\right) & \partial_{1} \frac{-g^{21}}{g^{22}}
\end{array}\right]\left[\begin{array}{l}
E_{3} \\
H_{1}
\end{array}\right] }
\end{aligned}
$$


TM polarization

$$
\begin{aligned}
\partial_{2}\left[\begin{array}{c}
H_{3} \\
E_{1}
\end{array}\right]= & {\left[\begin{array}{cc}
\frac{-g^{12}}{g^{22}} \partial_{1} & i \omega \epsilon_{0} \epsilon\left(x^{1}\right) \frac{1}{\sqrt{g} g^{22}} \\
i \omega \mu_{0}\left(\sqrt{g} g^{33}+\frac{1}{k^{2}} \partial_{1} \frac{1}{\epsilon\left(x^{1}\right) \sqrt{g} g^{22}} \partial_{1}\right) & \partial_{1} \frac{-g^{21}}{g^{22}}
\end{array}\right]\left[\begin{array}{l}
H_{3} \\
E_{1}
\end{array}\right] }
\end{aligned}
$$

Since the coefficients of the above operators do not depend on $x^{2}$, any component of the field may be written as:

$$
\psi\left(x^{1}, x^{2}\right)=\phi\left(x^{1}\right) \exp \left(-i k \beta x^{2}\right)
$$

which amounts to replace operator $\partial_{2}$ by $-i k \beta$. Hence, the systems of differential equations 15 and 16 become two eigenvalue equations which may be written as:

\section{TE polarization}

$$
\begin{aligned}
\beta\left[\begin{array}{l}
E_{3}\left(x^{1}\right) \\
H_{1}\left(x^{1}\right)
\end{array}\right]= & \\
& {\left[\begin{array}{cr}
-\frac{i}{k} \frac{g^{12}}{g^{22}} \partial_{1} & Z_{0} \frac{1}{\sqrt{g} g^{22}} \\
\frac{1}{Z_{0}}\left(\epsilon\left(x^{1}\right) \sqrt{g} g^{33}+\frac{1}{k^{2}} \partial_{1} \frac{1}{\sqrt{g} g^{22}} \partial_{1}\right) & -\frac{i}{k} \partial_{1} \frac{g^{21}}{g^{22}}
\end{array}\right]\left[\begin{array}{l}
E_{3}\left(x^{1}\right) \\
H_{1}\left(x^{1}\right)
\end{array}\right] }
\end{aligned}
$$

TM polarization

$$
\begin{aligned}
\beta\left[\begin{array}{l}
H_{3}\left(x^{1}\right) \\
E_{1}\left(x^{1}\right)
\end{array}\right]= & \\
& {\left[\begin{array}{cc}
-\frac{i}{k} \frac{g^{12}}{g^{22}} \partial_{1} & -\frac{1}{Z_{0}} \epsilon\left(x^{1}\right) \frac{1}{\sqrt{g} g^{22}} \\
Z_{0}\left(\sqrt{g} g^{33}+\frac{1}{k^{2}} \partial_{1} \frac{1}{\epsilon\left(x^{1}\right) \sqrt{g} g^{22}} \partial_{1}\right) & -\frac{i}{k} \partial_{1} \frac{g^{21}}{g^{22}}
\end{array}\right]\left[\begin{array}{l}
H_{3}\left(x^{1}\right) \\
E_{1}\left(x^{1}\right)
\end{array}\right] }
\end{aligned}
$$

where $Z_{0}$ is the vacuum impedance. Since we consider binary gratings, it has to be observed that operators $\epsilon\left(x^{1}\right) \partial_{1} \frac{1}{\epsilon\left(x^{1}\right)} \partial_{1}$ and $\partial_{1} \partial_{1}$ have the same form. In addition, we have $g=1, g^{22}=1$ and $g^{12}=-\tan \phi$. Hence, it is possible to write TE and TM matrix eigen-equations under the same form:

$$
\beta\left[\begin{array}{l}
F \\
G
\end{array}\right]=\left[\begin{array}{cc}
\tan \phi \frac{i}{k} \partial_{1} & 1 \\
\epsilon\left(x^{1}\right)+\frac{1}{k^{2}} \partial_{1} \partial_{1} & \tan \phi \frac{i}{k} \partial_{1}
\end{array}\right]\left[\begin{array}{l}
F \\
G
\end{array}\right]
$$

with

$$
T E \longrightarrow\left[\begin{array}{c}
F \\
G
\end{array}\right]=\left[\begin{array}{c}
E_{3} \\
Z_{0} H_{1}
\end{array}\right] \quad T M \longrightarrow\left[\begin{array}{c}
F \\
G
\end{array}\right]=\left[\begin{array}{c}
Z_{0} H_{3} \\
-\epsilon\left(x^{1}\right) E_{1}
\end{array}\right]
$$




\subsection{SOLUTION BY POLYNOMIAL EXPANSION}

\subsubsection{Polynomial expansion}

In this section, we solve numerically the spectral problem using the moment method with Legendre polynomials as expansion and test functions. We follow the very same lines which were previously used in ref [10] and in ref [12] In this approach, a period of the binary grating is first considered as a juxtaposition of homogeneous sub-domains in which we apply the moment method. In a second step, some boundary conditions have to be applied at the border of the sub-domains giving rise to a connection matrix. Indeed, the main difference between the present paper and the previous ones lies in the connection matrix. Because the coordinate system is non orthogonal, we have to enforce additional boundary conditions. In each region, the coordinate surfaces, $x_{1}^{1}=0, x_{2}^{1}=w$ and $x_{3}^{1}=d$ define two domains $\Omega_{1}$ and $\Omega_{2}$ in which the permittivity function is constant. Whatever the region, in the interval $x_{l}^{1} \leq x^{1} \leq x_{l+1}^{1}$ any function $\phi\left(x^{1}\right)$, its first derivative $\phi^{\prime}\left(x^{1}\right)$ and its second derivative $\phi^{\prime \prime}\left(x^{1}\right)$ are represented as:

$$
\left\{\begin{array}{l}
\phi^{(l)}\left(x^{1}\right)=\sum_{m} \phi_{m}^{(l)} P_{m}(\xi) \\
\phi^{\prime(l)}\left(x^{1}\right)=\sum_{m} \phi_{m}^{\prime(l)} P_{m}(\xi) \\
\phi^{\prime \prime(l)}\left(x^{1}\right)=\sum_{m} \phi^{\prime \prime}{ }_{m}^{(l)} P_{m}(\xi) \\
x_{l}^{1} \leq x^{1} \leq x_{l+1}^{1}, \quad l=1,2
\end{array}\right.
$$

the superscript $l$ refers to the domain. The reduced variable

$$
\xi=\frac{2 x^{1}-\left(x_{l+1}^{1}+x_{l}^{1}\right)}{\Delta^{(l)}}, \quad \Delta^{(l)}=x_{l+1}^{1}-x_{l}^{1}
$$

results from the mapping of the interval $\left[x_{l} x_{l+1}\right]$ onto the unit interval $[-11]$ on which the Legendre polynomials are orthogonal and complete:

$$
\int_{-1}^{1} P_{m}(\xi) P_{n}(\xi) d \xi=\frac{2}{2 m+1} \delta_{m n}
$$

where $P_{m}$ is the Legendre polynomial of degree $m$ and $\delta_{m n}$ designates the Kronecker symbol.

In order to get a matrix eigen-equation, we use the Galerkin method with Legendre polynomials as expansion and test functions. Since our problem consists of homogeneous domains, we shall get as many matrices as domains. However, a period of a binary grating is defined on two domains. So, in order to get the sought solution in terms of eigenmodes, we have to enforce some additional constraints to the expansion coefficients $\phi_{m}^{(l)}$ of functions $\phi^{(l)}(x)$. The observation of equation 20 shows that, apart from the identity operator, the only operators are first and second derivatives. 


\subsubsection{First and second derivative operators}

The derivative of $\phi^{(l)}$ with respect to $x$ is expanded on Legendre polynomials as:

$$
\frac{d \phi^{(l)}}{d x}=\frac{d \xi}{d x} \frac{d \phi}{d \xi}=\frac{2}{\Delta^{(l)}} \sum_{m} \phi_{m}^{(l)} \frac{d P_{m}(\xi)}{d \xi}=\sum_{m}{\phi^{\prime}}_{m}^{(l)} P_{m}(\xi)
$$

The Legendre polynomials and their derivatives satisfy:

$$
\int_{-1}^{1} \frac{d P_{m}(\xi)}{d \xi} P_{n}(\xi) d \xi= \begin{cases}2 & \text { for } \mathrm{m}-\mathrm{n}>0 \text { and } \mathrm{m}-\text { nodd } \\ 0 & \text { otherwise }\end{cases}
$$

which allows to link the coefficients $\phi_{n}^{(l)}$ of the function $\phi^{(l)}$ with the coefficients ${\phi_{m}^{\prime}}_{m}^{(l)}$ of its derivative $d \phi^{(l)} / d x$. Retaining $M^{(l)}$ coefficients in the derivative, we have

$$
\boldsymbol{\phi}_{m}^{(l)}=\mathbf{D}_{\mathbf{M}^{(1)}, \mathbf{M}^{(1)}+\mathbf{1}}^{(1)} \boldsymbol{\phi}_{\boldsymbol{n}}^{(l)} \quad m \in\left[0 M^{l}-1\right] \quad n \in\left[0 M^{(l)}\right]
$$

where $\mathbf{D}_{M^{(l)}, M^{(l)}+1}^{(l)}$ is a rectangular matrix with $M^{(l)}$ lines and $M^{(l)}+1$ columns whose non-null elements are the $D_{m n}^{(l)}$ such that:

$$
D_{m n}^{(l)}=\frac{\Delta^{(l)}}{2} \frac{2 m+1}{2}\left[1-(-1)^{n-m}\right] \quad n>m
$$

Let us now derive the matrix associated to the second derivative operator. Consider the column vector $\phi^{\prime \prime}{ }_{m}^{(l)}$ formed by the first $M^{l}$ expansion coefficients of the second order derivative of $\phi^{(l)}(x)$ on Legendre polynomials. We have:

$$
\phi{ }_{m}^{(l)}=\mathbf{D}_{2, M^{(l)}, M^{(l)}+2}^{(l)} \phi_{n}^{(l)}
$$

where

$$
\mathbf{D}_{2, M^{(l)}, M^{(l)}+2}^{(l)}=\mathbf{D}_{1, M^{l)}, M^{(l)}+1}^{(l)} \mathbf{D}_{1, M^{l)}+1, M^{(l)}+2}^{(l)}
$$

is a rectangular matrix with $M^{(l)}$ lines and $\left(M^{(l)}+2\right)$ columns. First and second derivative matrix operators are partitioned into the juxtaposition of a square matrix and a one column matrix and of a square matrix and a two column matrix respectively.

$$
\mathbf{D}_{1, M^{(l)}, M^{(l)}+1}^{(l)}=\left[\mathbf{D}_{1, S}^{(l)} \mathbf{D}_{1, C}^{(l)}\right], \quad \mathbf{D}_{2, M^{(l)}, M^{(l)}+2}^{(l)}=\left[\mathbf{D}_{2, S}^{(l)} \mathbf{D}_{2, C}^{(l)}\right]
$$

the subscripts $S$ and $C$ refer to the square part of the matrix and to the additional columns respectively.

\subsubsection{Connection matrix}

As is, the matrix associated to eigenvalue problem 20 is rectangular with more columns than rows. Whatever the polarisation, within each domain, we have two additional polynomial expansion coefficients for $F$ and one additional polynomial expansion coefficient for $G$. Hence, for the present problem with two 
domains, we need 6 more equations which can be easily obtained by enforcing the boundary conditions at $x=d$ and $x=w$. These boundary conditions are the continuity and the pseudo-periodicity of $H_{3}, D^{1}$ and $E_{2}$ for TM polarization and of $E_{3}, B^{1}$ and $H_{2}$ for TE polarization. They allow to derive the polarization dependent connection matrix $C_{p}$ such that

$$
\left[\begin{array}{l}
F_{M_{1}-1}^{(1)} \\
F_{M_{1}}^{(1)} \\
F_{M_{2}-1}^{(2)} \\
F_{M_{2}}^{(2)} \\
G_{M_{1}-1}^{(1)} \\
G_{M_{1}}^{(2)}
\end{array}\right]=\mathbf{C}_{\mathbf{p}}\left[\begin{array}{l}
F_{0}^{(1)} \\
\vdots \\
F_{M_{1}-2}^{(1)} \\
F_{0}^{(2)} \\
\vdots \\
F_{M_{2}-2}^{(2)} \\
G_{0}^{(1)} \\
\vdots \\
G_{M_{1}-2}^{(1)} \\
G_{0}^{(2)} \\
\vdots \\
G_{M_{2}-2}^{(2)}
\end{array}\right]
$$

Here, the subscript $p$ refers to the polarization.

\subsubsection{Matrix Operator}

The matrix associated to equation 20, is the sum of two matrices. One is the assembly of independent blocs which correspond to the sub-domains of the grating. The other is a full matrix that takes into account the transversal boundary conditions.

$$
\mathbf{L}=\mathbf{L}_{S}+\mathbf{L}_{C} \mathbf{C}_{p}
$$

The details of matrices $\mathbf{L}_{S}, \mathbf{L}_{C}$ and $\mathbf{C}_{\mathbf{p}}$ are given in appendix $\mathrm{A}$

\subsubsection{Summary}

The whole solution requires solving an eigenvalue problem for each region. It may seem surprising to numerically solve Maxwell's equations in homogeneous regions 1 and 3 where solutions are already known. The reason for doing so has been discussed in [12] and [15]. Hence, the solution in region 1 and 3 and consequently the computation of reflected and transmitted efficiencies is identical to what was done in[12]. The homogeneous regions and the grating regions are separated by coordinate surfaces $y=$ cte which are common to the Cartesian coordinate system and to the inclined coordinate system. So, matching the computed components $E_{3}, H_{1}$ and $H_{3}, E_{1}$ is straightforward. This is achieved by using the $\mathrm{S}$ matrix algorithm. The latter requires the separation of 
Table 1: Numerical comparison of efficiencies of a metallic grating calculated using the $\mathrm{C}$ method, DMM and the PMM.The number of plane waves and of modes in DMM is 60. In this paper we have $M^{(1)}=M^{(2)}=25$.

\begin{tabular}{llll}
\hline & \multicolumn{3}{l}{ TM polarization } \\
& C Method & \multicolumn{1}{c}{ DMM } & \multicolumn{1}{c}{ PMM } \\
\hline Diffraction Order & Efficiency & Efficiency & Efficiency \\
$R_{-1}$ & 0.2245 & 0.2247 & 0.2245 \\
$R_{0}$ & 0.3110 & 0.307 & 0.3113 \\
$T_{-1}$ & 0.2067 & 0.2075 & 0.2067 \\
$T_{0}$ & 0.2382 & 0.241 & 0.2381 \\
\hline
\end{tabular}

solutions into forward waves and backward waves. In order to make this sorting easy in the grating region, we always consider lossy materials. If necessary, we make the grating region lossy by adding a very small negative imaginary part to the dielectric constant. It is numerically observed that there are as many forward waves as backward waves. The forward (respectively backward) waves are those waves for which $\beta$ has a negative (respectively positive) imaginary part.

\section{Numerical results}

In this section, we validate our code by comparing our results with already published data which was obtained with the Differential Modal Method (DMM)in [8] and the $\mathrm{C}$ method in [6]. In fact, as outlined in [7], the factorization rules were not implemented correctly in [6] for TM Polarization. We corrected the problem, and the results obtained with $\mathrm{C}$-method given in the present paper are slightly different from those of $[6]$.

\subsection{Metallic grating}

The first case concerns a metallic grating with $d=1 \mu \mathrm{m}, f_{d}=0.5 \mu \mathrm{m}, h=$ $0.2 \mu \mathrm{m}$, slant angle $\phi=10^{\circ}$. The incident wavelength and incident angle are $\lambda=1 \mu \mathrm{m}$ and $\theta=30^{\circ}$, respectively. The optical indices are $\nu_{1}=1\left(\epsilon_{1}=1\right)$, $\left.\epsilon_{22}=1, \epsilon_{21}=(.22-i \times 6.71)^{2}=-44.9757-2.9524 i\right)$ and $\epsilon_{3}=1.45^{2}=2.1025$. Table 1 and table 2 give the numerical values obtained with the $\mathrm{C}$ method, the DMM, and the Polynomial Modal Method (PMM). It is observed that all results coincide remarkably well even though a very slight discrepancy occurs in TM polarization for DMM. For the same case, figures 2 and 3 illustrate the convergence of the reflected efficiencies in TM polarization. In order to better show how fast the numerical convergence is and also the stability of the method, the horizontal axis scales as the inverse of the truncation number which is the rank of the matrix from which eigenvectors are sought. It is seen that convergence is very fast and that no instabilities occur. 
Table 2: Numerical comparison of efficiencies of a metallic grating calculated Using the $\mathrm{C}$ method, DMM and the PMM. The number of plane waves and of modes in DMM is 50. In this paper we have $M^{(1)}=M^{(2)}=25$

\begin{tabular}{llll}
\hline & \multicolumn{2}{l}{ TE polarization } & \\
& C Method & DMM & PMM \\
\hline Diffraction Order & Efficiency & Efficiency & Efficiency \\
\hline$R_{-1}$ & 0.2358 & 0.2359 & 0.2358 \\
$R_{0}$ & 0.4268 & 0.4267 & 0.4268 \\
$T_{-1}$ & 0.1646 & 0.1646 & 0.1646 \\
$T_{0}$ & 0.1556 & 0.1557 & 0.1556 \\
\hline
\end{tabular}

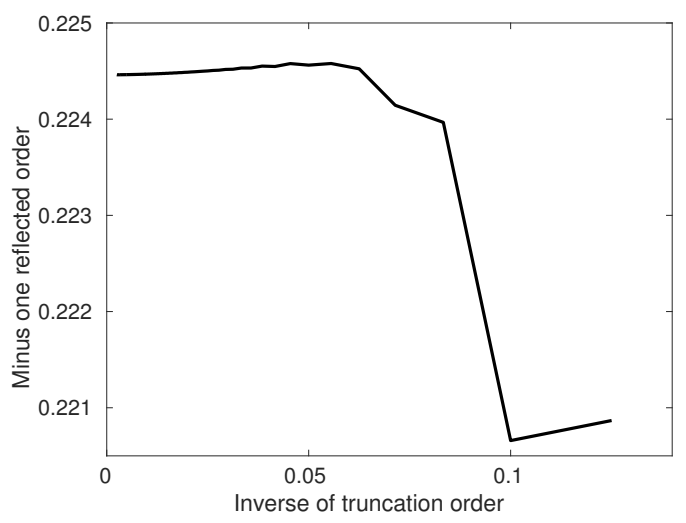

Figure 2: Convergence plot of the minus one reflected order of a metallic grating in TM polarization

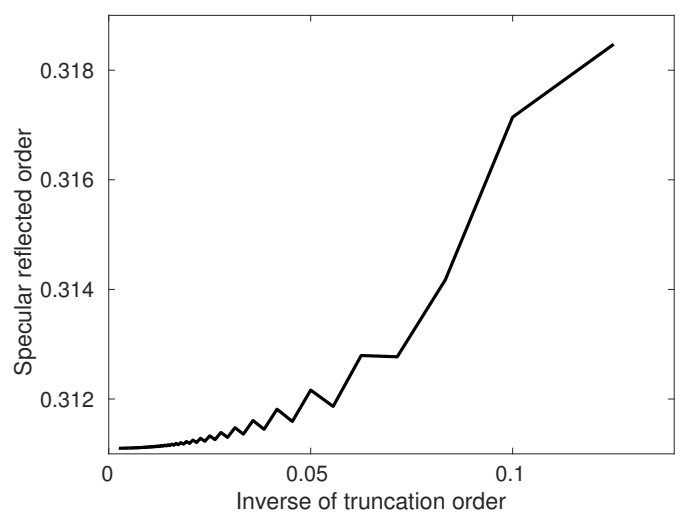

Figure 3: Convergence plot of the specular reflected order of a metallic grating in TM polarization 
Table 3: Numerical comparison of efficiencies of a dielectric grating calculated Using the $\mathrm{C}$ method, DMM and the PMM.The number of plane waves and of modes in DMM is 30. In this paper we have $M^{(1)}=M^{(2)}=14$

\begin{tabular}{llll}
\hline & \multicolumn{3}{l}{ TM polarization } \\
& C Method & DMM & PMM \\
\hline Diffraction Order & Efficiency & Efficiency & Efficiency \\
\hline$R_{-1}$ & 0.0231 & 0.0231 & 0.0231 \\
$R_{0}$ & 0.0011 & 0.0011 & 0.0011 \\
$T_{-1}$ & 0.0227 & 0.0227 & 0.0227 \\
$T_{0}$ & 0.9531 & 0.9531 & 0.9531 \\
\hline
\end{tabular}

Table 4: Numerical comparison of efficiencies of a dielectric grating calculated Using the $\mathrm{C}$ method,the DMM and the PMM.The number of plane waves and of modes in DMM is 30. In this paper we have $M^{(1)}=M^{(2)}=14$.

\begin{tabular}{llll}
\hline & \multicolumn{3}{l}{ TE polarization } \\
& C Method & DMM & PMM \\
\hline Diffraction Order & Efficiency & Efficiency & Efficiency \\
\hline$R_{-1}$ & 0.0179 & 0.0179 & 0.0179 \\
$R_{0}$ & 0.0137 & 0.0137 & 0.0137 \\
$T_{-1}$ & 0.0399 & 0.0398 & 0.0399 \\
$T_{0}$ & 0.9286 & 0.9286 & 0.9286 \\
\hline
\end{tabular}

\subsection{Dielectric grating}

Let us consider a dielectric grating with $d=1 \mu \mathrm{m}, f_{d}=0.5 \mu \mathrm{m}, h=0.2 \mu \mathrm{m}$, slant angle $\phi=10^{\circ}$. The incident wavelength and incident angle are $\lambda=1 \mu \mathrm{m}$ and $\theta=30^{\circ}$, respectively. The optical indices are $\nu_{1}=1\left(\epsilon_{1}=1\right), \epsilon_{22}=2.25$ and $\epsilon_{3}=1.45^{2}=2.1025$. Table 3 and table 4 give the numerical values obtained with the $\mathrm{C}$ method,the DMM, and the PMM. Once more, all three methods give the same values.

\subsection{Large slant angle}

In [8], Campbell et al investigated the case where the slant angle becomes higher and higher and tends to $90^{\circ}$. In that case, they found that the grating behaves as a multilayer stack with layers thinner and thinner. At the limit, when the slant angle tends to $90^{\circ}$, the grating can be considered as a slab with a premittivity equal to the average of the permittivities of the gratings. At normal incidence, the reflected and transmitted energy should be the same for TE and TM polarizations. The plots in figures 4 and 6 reproduce the results obtained by Campbell et al and shown in Figures 5 and 6 of [8]. For the investigated cases, we have sketched that the reflected efficiency computed with the largest possible slant angle -that is $89.99^{\circ}$ coincides with that of the corresponding ef- 
fective slab. It has to be noted that Campbell et al did not provide figure 5 . They commented they had convergence problem for that case.

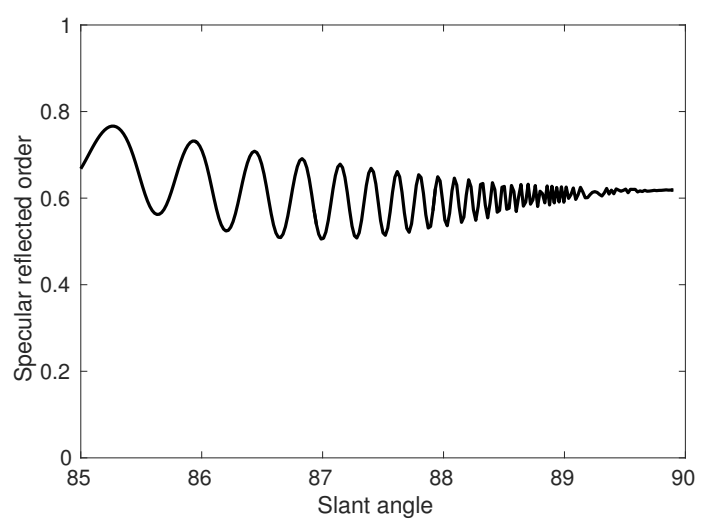

Figure 4: Specular reflected order versus slant angle for a dielectric grating enlightened under normal incidence in TE polarization. $w=0.5, d=1, h=0.5$, $\lambda=1.1, \epsilon_{21}=1, \epsilon_{22}=25-i 0.0001$.The grating is suspended in air. The effective permittity is 13 . The reflectivity of the corresponding effective slab is 0.6191 . We obtain the same value with a slant angle of $89.99^{\circ}$

\section{Conclusion}

In this paper, we extended the polynomial modal method to the case of slanted lamellar gratings. The formulation of the problem relies on the use of an inclined coordinate system and of Maxwell's equations in covariant form. The novelty of our work lies in the numerical solution. Solving the problem with polynomial expansions amounts to using a multi-domain approach. The matrix from which eigenvectors and eigenvalues are sought is the sum of a bloc diagonal matrix independent of polarization and a full connection matrix that takes into account polarization and boundary conditions at the border of the sub-domains. We have successfully compared our new numerical method to previously published data especially those of [8]. In particular, we were able to deal with slant angle as high as $89.9^{\circ}$ without facing neither convergence problem nor numerical instabilities. In the future we will implement the method in the case of conical incidence.

\section{Appendix A}

In this appendix, we detail the construction of the connection matrix $C_{p}$. In order to compute the solution of eigen value problem of, we have to enforce some boundary conditions at coordinate surface $x^{1}=w$ and $x=d$. First of all, 


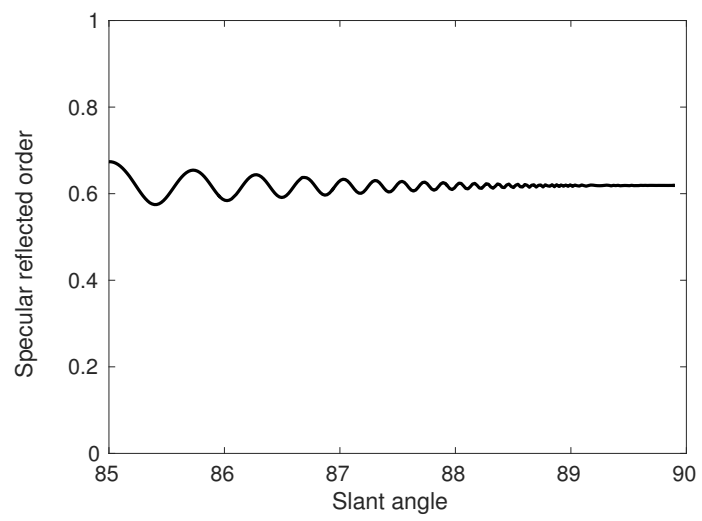

Figure 5: Specular reflected order versus slant angle for a dielectric grating enlightened under normal incidence in TM polarization. $w=0.5, d=1, h=0.5$, $\lambda=1.1, \epsilon_{21}=1, \epsilon_{22}=25-i 0.0001$. The grating is suspended in air. The effective permittity is 13 . The reflectivity of the corresponding effective slab is 0.6191 . We obtain the same value with a slant angle of $89.99^{\circ}$

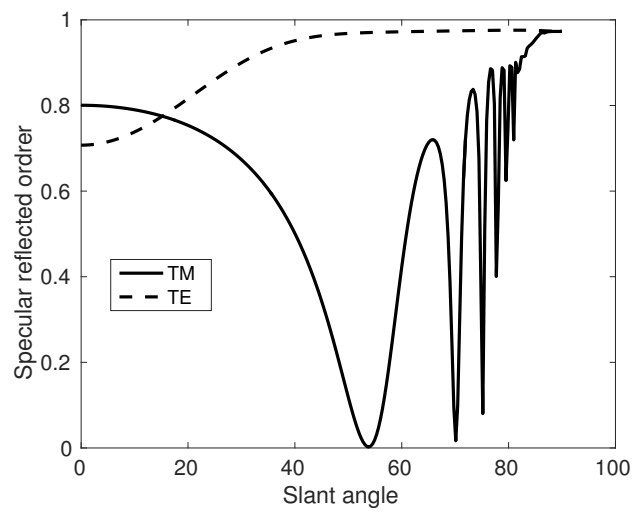

Figure 6: Specular reflected order versus slant angle for a dielectric grating enlightened under normal incidence in TE polarization and in TM polarization. $w=0.5, d=1, h=0.5, \lambda=1.1, \epsilon_{21}=1, \epsilon_{22}=(0.22-i * 6.71)^{2}$. The grating is suspended in air. The effective permittity is $-21.9878-i \times 1.4762$. The reflectivity of the corresponding effective slab is 0.9731 . We obtain the same value with a slant angle of $89.99^{\circ}$ 
the field is pseudo periodic which means that every field component satisfies :

$$
U\left(x^{1}+d\right)=\tau U\left(x^{1}\right)
$$

where the coefficient $\tau$ is given by:

$$
\tau=\exp \left(-i k \alpha_{0} d\right)
$$

At $x^{1}=w, D^{1}, H_{3}$ and $E_{2}$ are continuous in TE polarization, as well as $B^{1}$, $E_{3}$ and $H_{2}$ in TM polarization. Using polynomial expansions allows to enforce in an exact manner these boundary conditions. From Maxwell's equations we may derive the following expressions:

$$
\begin{gathered}
D^{1}=-\frac{g^{12}}{g^{22}} \partial_{1} H_{3}+i \omega \epsilon_{0} \epsilon\left(x^{1}\right) \frac{1}{\sqrt{g} g^{22}} E_{1} \\
E_{2}=\frac{i}{\omega \epsilon_{0} \epsilon\left(x^{1}\right)} \partial_{1} H_{3}-\frac{g^{21}}{g^{22}} E_{1} \\
B^{1}=-\frac{g^{12}}{g^{22}} \partial_{1} E_{3}-i \omega \mu_{0} \frac{1}{\sqrt{g} g^{22}} H_{1} \\
H_{2}=-\frac{i}{\omega \mu_{0}} \partial_{1} E_{3}-\frac{g^{21}}{g^{22}} H_{1}
\end{gathered}
$$

The above expressions may be re-written in terms of $F$ and $G$. For TM polarization we have :

$$
\begin{gathered}
Z_{0} D^{1}=-\frac{g^{12}}{g^{22}} \partial_{1} F-i k \frac{1}{\sqrt{g} g^{22}} G \\
E_{2}=\frac{1}{\epsilon\left(x^{1}\right)}\left(\frac{i}{k} \partial_{1} F-\frac{g^{21}}{g^{22}} G\right)
\end{gathered}
$$

and for TE we have:

$$
\begin{gathered}
B^{1}=-\frac{g^{12}}{g^{22}} \partial_{1} F-i k \frac{1}{\sqrt{g} g^{22}} G \\
Z_{0} H_{2}=-\frac{i}{k} \partial_{1} F-\frac{g^{21}}{g^{22}} G
\end{gathered}
$$

Although we have put the TE and TM eigen equations under the same form by introducing variable $F$ and $G$, the solutions differ because $F$ and $G$ do not satisfy the same boundary conditions. Indeed as is shown by relations 41 and 43 , the continuity of $E_{2}$ for TM polarization and the continuity of $Z_{0} H_{2}$ for TE polarization involve $F$ and $G$ differently. In order to have a single expression for 41 and 43 let us introduce $\kappa_{f}, \kappa_{g}$ such that: 


$$
\begin{gathered}
\kappa_{f}= \begin{cases}\frac{1}{\epsilon} & \text { for TM polarization } \\
-1 & \text { for TE polarization }\end{cases} \\
\kappa_{g}= \begin{cases}\frac{1}{\epsilon} & \text { for TM polarization } \\
1 & \text { for TE polarization }\end{cases}
\end{gathered}
$$

Then 41 as well as 43 reads:

$$
E_{2}=\frac{i}{k} \kappa_{f} F-\frac{g^{21}}{g^{22}} \kappa_{g} G
$$

The Legendre polynomials $P_{m}$ satisfy:

$$
P_{m}( \pm 1)=( \pm 1)^{m} \quad P_{m}^{\prime}( \pm 1)=( \pm 1)^{m+1} \frac{m(m+1)}{2}
$$

where $P_{m}^{\prime}$ denotes the derivative of $P_{m}$. Every region consists of the two domains $\Omega_{1}$ and $\Omega_{2}$ such that $\Omega_{1}=\left\{x^{1}, 0 \leq x^{1} \leq w\right\}$ and $\Omega_{2}=\left\{x^{1}, 0 \leq x^{1} \leq w\right\}$. We refer to these two domains by the superscripts (1) and (2) respectively. Lastly, let us introduce $d_{+m}^{(1)}, d_{-m}^{(1)}, d_{+m}^{(2)}$ and $d_{-m}^{(2)}$ defined by:

$$
\begin{array}{cc}
d_{+m}^{(1)}=\frac{1}{w} \frac{m(m+1)}{2} & d_{-m}^{(1)}=(-1)^{m} \frac{1}{w} \frac{m(m+1)}{2} \\
d_{+m}^{(2)}=\frac{1}{d-w} \frac{m(m+1)}{2} & d_{-m}^{(2)}=(-1)^{m} \frac{1}{d-w} \frac{m(m+1)}{2}
\end{array}
$$

Taking into account $g^{22}=1, g^{21}=-\tan \phi$, we are now ready to write the six boundary conditions at $x^{1}=w$ and $x^{1}=d$ :

- Continuity of F

$$
\sum_{m=0}^{M(1)+1} F_{m}^{(1)}-\sum_{m=0}^{M(2)+1}(-1)^{m} F_{m}^{(2)}=0
$$

- Pseudoperiodicity of $\mathrm{F}$

$$
\sum_{m=0}^{M(1)+1} F_{m}^{(2)}-\tau \sum_{m=0}^{M(1)+1}(-1)^{m} F_{m}^{(1)}=0
$$

- Continuity of $\frac{i}{k} \kappa_{f} \partial_{1} F+\tan \phi \kappa_{g} G$

$$
\begin{array}{r}
\kappa_{f}^{(1)} \frac{i}{k} \sum_{m=0}^{M(1)+1} d_{+m}^{(1)} F_{m}^{(1)}+\kappa_{g}^{(1)} \tan \phi \sum_{m=0}^{M(1)} G_{m}^{(1)} \\
-\kappa_{f}^{(2)} \frac{i}{k} \sum_{m=0}^{M(2)+1} d_{-m}^{(2)} F_{m}^{(2)}-(-1)^{m} \kappa_{g}^{(2)} \tan \phi \sum_{m=0}^{M(1)} G_{m}^{(1)} \\
=0
\end{array}
$$


- pseudo periodicity of $\frac{i}{k} \kappa_{f} F+\tan \phi \kappa_{g} G$

$$
\begin{array}{r}
\kappa_{f}^{(2)} \frac{i}{k} \sum_{m=0}^{M(1)+1} d_{+m}^{(2)} F_{m}^{(2)}+\kappa_{g}^{(2)} \tan \phi \sum_{m=0}^{M(1)} G_{m}^{(2)} \\
-\tau \kappa_{f}^{(1)} \frac{i}{k} \sum_{m=0}^{M(1)+1} d_{-m}^{(1)} F_{m}^{(1)}-\tau(-1)^{m} \kappa_{g}^{(1)} \tan \phi \sum_{m=0}^{M(1)} G_{m}^{(1)} \\
=0
\end{array}
$$

- continuity of $\tan \phi \partial_{1} F-i k G$

$$
\begin{array}{r}
\tan \phi \sum_{m=0}^{M(1)+1} d_{+m}^{(1)} F_{m}^{(1)}-i k \sum_{m=0}^{M(1)} G_{m}^{(1)} \\
-\tan \phi \sum_{m=0}^{M(2)+1} d_{-m}^{(2)} F_{m}^{(2)}+(-1)^{m} i k \sum_{m=0}^{M(2)} G_{m}^{(2)}=0
\end{array}
$$

- pseudo periodicity of $\tan \phi \partial_{1} F-i k G$

$$
\begin{array}{r}
\tan \phi \sum_{m=0}^{M(1)+1} d_{+m}^{(2)} F_{m}^{(2)}-i k \sum_{m=0}^{M(2)} G_{m}^{(2)} \\
-\tau \tan \phi \sum_{m=0}^{M(1)+1} d_{-m}^{(1)} F_{m}^{(1)}+\tau(-1)^{m} i k \sum_{m=0}^{M(1)} G_{m}^{(1)}=0
\end{array}
$$

The derivation of matrix $C p$ from 49, 50, 51, 5253 and 54 is straightforward Finally matrices $\boldsymbol{L}_{S}$ and $\boldsymbol{L}_{C}$ write:

$$
\begin{gathered}
\boldsymbol{L}_{S}= \\
{\left[\begin{array}{cccc}
\frac{i}{k} \tan \phi \mathbf{D}_{1, S}^{(1)} & \ldots & \mathbf{I}^{(1)} & \ldots \\
\ldots & \frac{i}{k} \tan \phi \mathbf{D}_{1, S}^{(2)} & \ldots & \mathbf{I}^{(2)} \\
\epsilon^{(1)} \mathbf{I}^{(1)}+\mathbf{D}_{2, S}^{(1)} & \ldots & \frac{i}{k} \tan \phi \mathbf{D}_{1, S}^{(1)} & \ldots \\
\ldots & \epsilon^{(2)} \mathbf{I}^{(2)}+\mathbf{D}_{2, S}^{(2)} & \ldots & \frac{i}{k} \tan \phi \mathbf{D}_{1, S}^{(2)}
\end{array}\right]} \\
\boldsymbol{L}_{C}=\left[\begin{array}{cccc}
\frac{i}{k} \tan \phi \tilde{\mathbf{D}}_{1, C}^{(1)} & \ldots & \ldots & \ldots \\
\ldots & \frac{i}{k} \tan \phi \tilde{\mathbf{D}}_{1, C}^{(2)} & \ldots & \ldots \\
\frac{1}{k^{2}} \boldsymbol{D}_{2, C}^{(1)} & \ldots & \frac{i}{k} \tan \phi \boldsymbol{D}_{1, C}^{(1)} & \ldots \\
\ldots & \frac{1}{k^{2}} \boldsymbol{D}_{2, C}^{(2)} & \ldots & \frac{i}{k} \boldsymbol{D}_{1, C}^{(2)}
\end{array}\right]
\end{gathered}
$$


For notation convenience we have introduced the matrix:

$$
\tilde{\boldsymbol{D}}_{1 C}=\left[\boldsymbol{D}_{1 C} \mathbf{0}\right]
$$

which is the juxtaposition of the one column matrix $\boldsymbol{D}_{1 C}$ and of a one column matrix filled with zeros.

\section{References}

[1] Petit R, 1980 Electromagnetic Theory of Grating Springer-Verlag, Berlin;

[2] Nevière M and Popov E 2003Light Propagation in Periodic Media Marcel Dekker, inc.

[3] Moharam M G, Grann E. B., Pommet D. A. Pommet, and Gaylord T. K. 1995 "Formulation for stable and efficient implementation of the rigorous coupled-wave analysis of binary gratings," J.Opt. Soc. Am. A12, 1068-1076.

[4] Moharam M G, and Gaylord T K, 1981 "Rigorous coupled-wave analysis of planar-grating diffraction," J.Opt. Soc. Am. A 71, 811-818.

[5] E. Popov, M. Nevière,2000, J. Opt. Soc. Am. A17 1773-1784.

[6] Granet G, Chandezon J, and Coudert O, 1997 "Extension of the C method to nonhomogeneous media: application to nonhomogeneous layer with parallel modulated faces and to inclined lamellar gratings,"J.Opt. Soc. Am. A14 $1576-1582$

[7] Li L "Oblique-coordinate system based Chandezon method for modelling one-dimensional periodic,multilayer,inhomgeneous,anisotropic gratings", 1999 J.Opt.Soc.Am.A 16, 2521-2531

[8] Campbell S, Botten L C, Mcphedran R C, and Martijn de Sterke C, 2008 " Modal method for classical diffraction by slanded lamellar gratings," J.Opt. Soc. Am. A25 2415-2426.

[9] Morf R H,1995 "Exponentially convergent and numerically efficient solution of Maxwell's equations for lamellar gratings", J. Opt. Am. A 12 1043-1056

[10] Edee K,2011 "Modal method based on sub-sectional Gegenbauer polynomial expansion for lamellar grating" J. Opt. Soc. Am A28, 2006-2013.

[11] Edee K, Fenniche I, Granet G and Guizal B,2013 "Modal method based on sub-sectional Gegenbauer polynomial expansion for lamellar gratings:weighting function, convergence and stability",Progress In Electromagnetic Research, 133, 17-35.

[12] Randriamihaja M H, Granet G, Edee K, Raniriharinosy K,2016 "Polynomial modal analysis of lamellar diffraction gratings in conical mounting" J. Opt. Soc. Am A33,1679-1686 
[13] Chandezon J, Maystre D and Raoult G 1980 J.Optique 11 235-41

[14] Chandezon J, Dupuis M T, Cornet G and Maystre D 1982 J.Opt.Soc. Am. $72839-46$

[15] G. Granet, "Fourier-matching pseudospectral modal method for diffraction gratings: comment," 2012 J. Opt. Soc. Am. A 29 1843-1845. 